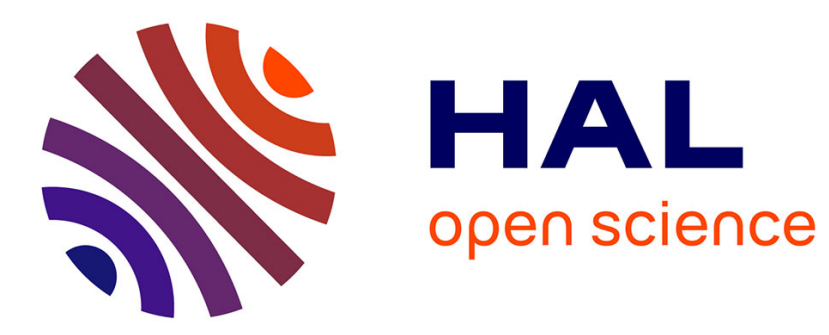

\title{
L'utilisation de plots de forme trapézoïdale dans les machines à reluctance variable
}

\author{
J.P. Bastos
}

\section{To cite this version:}

J.P. Bastos. L'utilisation de plots de forme trapézoïdale dans les machines à reluctance variable. Revue de Physique Appliquée, 1982, 17 (10), pp.693-700. 10.1051/rphysap:019820017010069300 . jpa-00245047

\section{HAL Id: jpa-00245047 https://hal.science/jpa-00245047}

Submitted on 1 Jan 1982

HAL is a multi-disciplinary open access archive for the deposit and dissemination of scientific research documents, whether they are published or not. The documents may come from teaching and research institutions in France or abroad, or from public or private research centers.
L'archive ouverte pluridisciplinaire HAL, est destinée au dépôt et à la diffusion de documents scientifiques de niveau recherche, publiés ou non, émanant des établissements d'enseignement et de recherche français ou étrangers, des laboratoires publics ou privés. 


\title{
L'utilisation de plots de forme trapézoïdale dans les machines à reluctance variable
}

\author{
J. P. Bastos \\ Laboratoire d'Electrotechnique, Université de Paris XI, Bât. 214, 91405 Orsay Cedex, France
}

(Reçu le 18 novembre 1981, révisé le 16 mars 1982, accepté le 7 juin 1982)

\begin{abstract}
Résumé. - L'étude concerne un type particulier de machine à hautes performances : la machine à réluctance variable à disques imbriqués. Suite à une première étude, traitant des plots de forme rectangulaire classique, nous présentons celle-ci, où l'on montre que l'utilisation de plots ayant une forme trapézoïdale est susceptible d'améliorer de façon significative les performances de ces machines. Les résultats sont aussi applicables à d'autres types de machines à réluctance variable.
\end{abstract}

\begin{abstract}
This study concerns a kind of high performance machine : the multi-interleaved disks variable reluctance machine. In the paper, we show that the use of trapezoidal shape for the plots, instead of the classical rectangular one, is able to increase the machine performance. The results of the study can also be applied to other kind of reluctance machines.
\end{abstract}

\section{Liste des principales grandeurs}

H champ magnétique.

B induction magnétique.

$\mu \quad$ perméabilité magnétique.

$\Phi$ flux magnétique.

$V$ potentiel scalaire magnétique.

$v \quad$ vitesse de défilement des plots.

$H_{\text {c }}$ valeur du champ magnétique au "coude» de saturation du matériau magnétique.

h champ magnétique en unité réduite.

$\mu_{\mathrm{r}} \quad$ perméabilité relative.

${ }_{\mathcal{F}}$ flux de l'induction magnétique en unité réduite.

৩ potentiel scalaire magnétique en unité réduite.

$P$ puissance volumique.

$P_{\mathrm{T}}$ pression tangentielle moyenne sur un cycle.

$l_{1}, l_{2}, l_{3}, L, E$ dimensions du domaine élémentaire (Fig. 2c).

Paramètres de forme

$e=E / L$ entrefer relatif.

$\lambda=l_{1} / L$ forme extérieure du domaine.

$s=l_{2} / l_{1}$ proportion de fer.

$t=l_{3} / l_{2}$ définition de l'inclinaison du plot.

1. Introduction. - Cette étude fait suite à celle référencée en [3]. Elle concerne une machine électrique à hautes performances : la machine à réluctance variable à disques imbriqués [1,2].

Rappelons d'abord le fonctionnement de cette machine, dont la présentation se trouve sur la figure 1 :

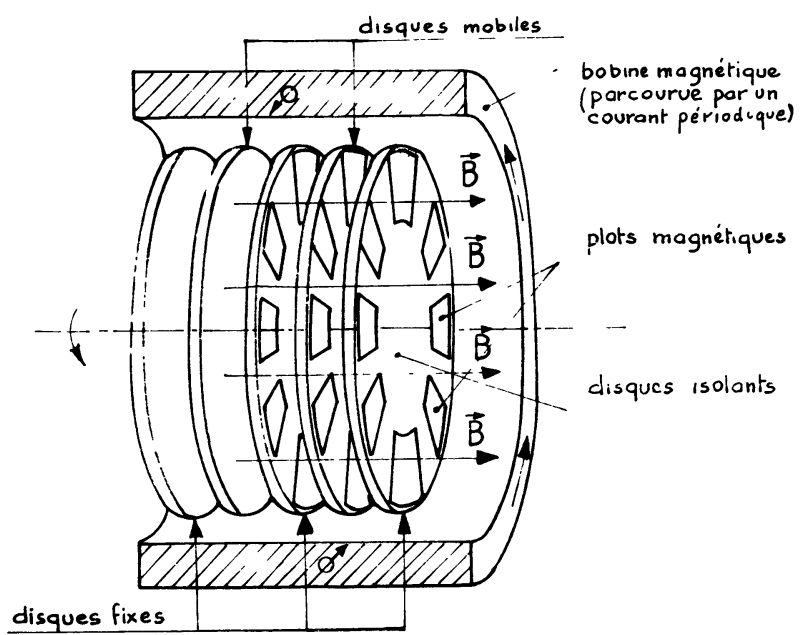

Fig. 1. - Structure d'une machine à disques imbriqués.

[Structure of an interleaved disks machine.]

on distingue deux séries de disques alternativement fixes et mobiles. L'ensemble est disposé à l'intérieur d'un bobinage inducteur, créant un champ d'induction global B. Les disques, en matière isolante non magnétique, possèdent un grand nombre de petits plots ferro-magnétiques. Tous les plots de disques fixes sont en face les uns des autres. Il en est de même pour ceux des disques mobiles. La variation de réluctance du circuit est obtenue lors de la rotation des disques mobiles. Cette réluctance est minimale lorsque tous les plots sont alignés ou en "conjonction». Elle est 
maximale lorsque ils sont décalés ou en " opposition ". Nous étudions la machine à partir d'un cycle dit idéal et qui correspond au fonctionnement moteur suivant : le courant inducteur croît rapidement lorsque les plots sont en opposition. On maintient ensuite le courant constant pendant le passage des plots à la conjonction suivante. A cette position on impose alors une brusque décroissance du courant (cf. [3]). L'énergie cinétique des disques les conduit enfin à une nouvelle opposition et le cycle recommence.

L'étude globale de ces machines, dans notre laboratoire, comporte trois axes principaux de recherche :

- la détermination numérique chiffrée de leurs performances, en tenant compte du régime de saturation des plots;

- l'étude conceptuelle globale de la machine traitant du dimensionnement de ses différentes parties ;

- l'étude de l'alimentation et du pilotage de la machine.

Dans le cadre de cet article nous étudions le premier point.

L'étude concerne uniquement la région utile des disques qui crée la variation de réluctance. Les performances envisagées font, donc, abstraction des dispositifs extérieurs aux disques fixes et mobiles (bobinages inducteurs, circuits de retour du flux magnétique, source de courant, etc...) et des pertes qui y sont engendrées.

Ce problème a déjà été abordé en [3]; nous avons considéré que la section des plots, dans le sens axial, est rectangulaire. Nous avons alors dégagé les performances correspondant à de très nombreuses configurations de plots et une optimisation morphologique a été faite. Par ailleurs, dans une étude préliminaire [4], nous avons remarqué que l'utilisation des plots ayant des sections trapézoïdales était susceptible d'améliorer nettement les performances de la machine. Les résultats présentés ici complètent cette étude de façon systématique.

Nous rappelons, en préliminaire, les principales grandeurs physiques intervenant dans la présente étude et déjà utilisées dans [3].

2. Modèle numérique. -2.1 LE DOMAINE ÉLÉMENTAIRE. - La géométrie du problème est tridimensionnelle, ce qui le rend délicat à résoudre. Nous avons donc étudié un modèle approché en négligeant la dépendance radiale. Le problème a été ainsi ramené à celui de la figure $2 a$, qui par suite des conditions de périodicité, se réduit à celui du " domaine élémentaire " de la figure $2 b$, définissant ses dimensions. La forme géométrique du domaine est représentée alors par les paramètres suivants :

$$
\begin{aligned}
& e=E / L \text { (entrefer relatif) } \\
& \lambda=l_{1} / L \text { (forme extérieure) } \\
& s=l_{2} / l_{1} \quad \text { (proportion de fer) } .
\end{aligned}
$$

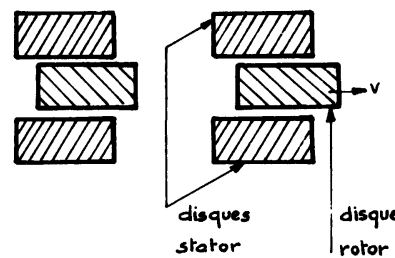

Fig. 2a. - Développement bidimensionnel des surfaces d'interaction.

[Bidimensional projection of interaction surfaces.]

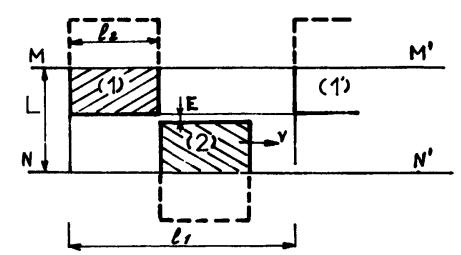

Fig. 2b. - Domaine élémentaire adopté sur le modèle numérique.

[Shape of a elementary domain adopted in the numerical model.]

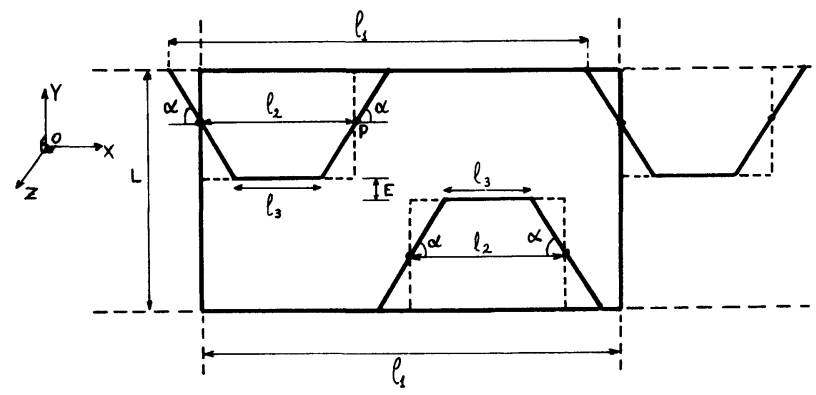

Fig. 2c. - Domaine élémentaire ayant des plots trapézoïdaux.

[Elementary domain with trapezoidals slots.]

On incline d'un angle $\alpha$ une face latérale du plot (Fig. $2 c$ ) autour de l'axe $\mathrm{O} Z$ perpendiculaire au plan de la figure et passant par le point $P$. Les plots trapézoïdaux, ainsi créés, sont définis par le paramètre supplémentaire

$$
t=l_{3} / l_{2} \text {. }
$$

La valeur $t=1$ correspond au plot rectangulaire. On remarque aussi, que $l_{2}$ étant pris à mi-hauteur du plot, la quantité de fer dans le domaine ne dépend que du paramètre $« s »$.

2.2 CyCle De FonCtionNement. - Lorsque les plots sont en opposition, le courant inducteur crée une différence de potentiel magnétique (DDP) $I_{0}$ entre les lignes équipotentielles $\mathbf{M M}^{\prime}$ et $\mathrm{NN}^{\prime}$ de la figure $2 b$. Celle-ci engendre un flux magnétique $\Phi$ sur la largeur $l_{1}$ et sur une profondeur unité suivant $\mathrm{OZ}$. Le courant est interrompu lors de la conjonction. Le cycle idéal 
étudié est représenté sur la figure 3 , où l'on met en évidence, dans le plan $\Phi, I$ la surface hachurée

$$
W=\int_{\text {cycle }} I \mathrm{~d} \Phi
$$

représentant de l'énergie recueillie sur un cycle dans le volume $l_{1} \times L \times 1$.

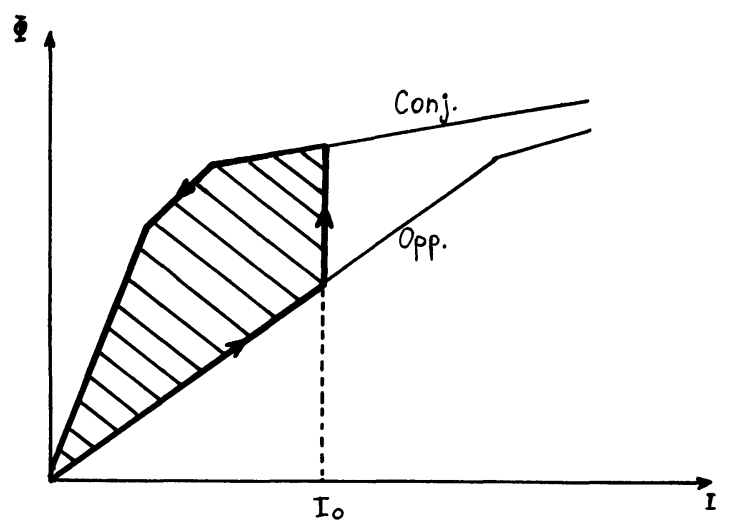

Fig. 3. - Cycle de fonctionnement dans le plan fluxampères tours.

[Work cycle in the flux-ampere turn plan.]

2. 3 L'ÉQUATION À RÉSOUDRE. - Dans le milieu où se trouvent les plots, nous avons les équations de la magnétostatique :

$$
\begin{array}{r}
\operatorname{rot} \mathbf{H}=0 \\
\operatorname{div} \mathbf{B}=0
\end{array}
$$

où $\mathbf{H}$ et $\mathbf{B}$ sont respectivement le champ et l'induction magnétique. $\mu$ étant la perméabilité magnétique, nous avons

$$
\mathbf{B}=\mu \mathbf{H} \quad \text { où } \quad \mu=\mu(|H|)
$$

comme il n'y a pas de courant dans le domaine, on utilise le potentiel pseudo-scalaire $V$, dont on dérive le champ magnétique $\mathbf{H}$. On a

$$
\begin{aligned}
\mathbf{H} & =-\operatorname{grad} V \\
\nabla \cdot \mathbf{B} & =\boldsymbol{\nabla} \cdot(\mu \mathbf{H})=-\nabla \cdot(\mu \nabla V)=0
\end{aligned}
$$

soit

$$
\frac{\partial}{\partial X}\left(\mu \frac{\partial V}{\partial X}\right)+\frac{\partial}{\partial Y}\left(\mu \frac{\partial V}{\partial Y}\right)=0
$$

Notre système informatique utilise une méthode variationnelle par éléments finis $[5,6]$ et il est décrit en [7]. Sa mise en œuvre nécessite l'introduction du maillage définissant le domaine, les conditions aux limites et des caractéristiques magnétiques du fer; le programme calcule ensuite les potentiels aux nouds, le champ et l'induction dans chaque élément. On déduit, alors, le flux traversant le domaine, et l'on atteint ensuite, à l'aide des diagrammes tracés, les performances de la machine.

Nous avons utilisés des grandeurs réduites ce qui permet une meilleure généralisation des résultats obtenus.

2.3.1. Fonction de perméabilité. - Le champ utilisé dans le calcul est $\mathrm{h}=\mathrm{H} / H_{\mathrm{c}}, H_{\mathrm{c}}$ étant le champ au " coude de saturation », et $\mathbf{H}$ le champ réel.

On a :

$|h|<1$ régime non saturé,

$|h| \geqslant 1$ régime de saturation.

Pour la fonction de perméabilité relative nous avons choisi pour

$$
\begin{array}{ll}
|h| \leqslant 1 & \mu_{\mathrm{r}}(h)=\mu_{\mathrm{r}}(h=0) \mathrm{e}^{-G h^{3} / 3} \\
|h| \geqslant 1 & \mu_{\mathrm{r}}(h)=1+\frac{\mu_{\mathrm{r}}(h=1)-1}{h}
\end{array}
$$

où $\mu_{\mathrm{r}}(h=0)$ et $G$ sont caractéristiques du matériau. Nous avons choisi : $\mu_{\mathrm{r}}(h=0)=1000$ et $G=0,9986$.

Le champ $\mathbf{H}=\mathbf{h} H_{\mathrm{c}}$ donne l'induction

$$
\begin{aligned}
& \mathbf{B}=\mu_{0} \mu_{\mathrm{r}} \mathbf{h} H_{\mathrm{c}} \text { où } \mu_{0}=4 \pi \times 10^{-7} \mathrm{H} \cdot \mathrm{m}^{-1} . \\
& \text { Lorsque } h=1 \text { on a }: B=B_{\text {saturation }}=B_{\mathrm{s}} \\
& B_{\mathrm{s}}=\mu_{0} \mu_{\mathrm{r}}(h=1) H_{\mathrm{c}}=\mu_{0} \mu_{\mathrm{r}}(h=0) \mathrm{e}^{-G / 3} H_{\mathrm{c}}
\end{aligned}
$$

soit aussi

$$
H_{\mathrm{c}}=B_{\mathrm{s}} / 7,17 \mu_{0} \text {. }
$$

Les calculs ont été exploités avec une valeur $B_{\mathrm{s}}=2,3 \mathrm{~T}$, donnant des courbes $\boldsymbol{B}(\boldsymbol{H})$ proches de celles obtenues pour des matériaux du type fer cobalt. Le choix de l'induction $B_{\mathrm{s}}$ fixe l'allure définitive de la courbe $B(H)$; il permet de passer des flux et des DDP calculés aux grandeurs réelles ( $\Phi$ et $I$ ). Ce passage est explicité au paragraphe suivant. Les résultats du calcul peuvent être, plus généralement, utilisés avec d'autres matériaux, pour lesquels $B_{\mathrm{s}}$ est différent de $2,3 \mathrm{~T}$. Il suffit pour cela que les courbes $B(H)$ simulées s'approchent suffisamment des courbes réelles. Notons à ce sujet, que du fait de la présence de l'entrefer, cette correspondance a surtout besoin d'être précise lorsque la perméabilité du fer est inférieure, en gros, à 800 . On constate, en effet, que, lorsque celle-ci est supérieure à $\mu_{\mathrm{r}}=800$ la réluctance du domaine ne dépend que des dimensions de l'entrefer. Ce n'est qu'au-dessous de cette valeur de $\mu_{\mathrm{r}}=800$ que le fer commence à s'opposer suffisamment au passage du champ pour que les courbes $(\Phi, I)$ cessent d'être linéaires.

Nous avons donc utilisé cette propriété en prenant une perméabilité $\mu_{\mathrm{r}}(h=0)=1000$, relativement plus faible que sur les matériaux réels ; ceci permet d'améliorer la convergence des calculs au voisinage du coude de saturation [8].

2.3.2 Courant, flux, travail par cycle, puissance volumique, pression tangentielle. - Dans le calcul sur 
ordinateur, on divise les dimensions de la figure $2 c$ par $L$. Les cotes $E, L, l_{1}$ deviennent alors $e, 1, \lambda$.

Nous avons donc :

$$
\mho=\int_{0}^{1} h_{y} \mathrm{~d} y \text { et } \mathcal{F}=\int_{0}^{\lambda} \mu_{\mathrm{r}} h_{y} \mathrm{~d} x
$$

$x$ et $y$ sont les coordonnées réduites du domaine élémentaire du calcul. Elles sont liées aux coordonnées réelles $X$ et $Y$ par les relations $Y=L y$ et $X=L x$. On a alors :

$$
\begin{gathered}
I=\int_{0}^{L} H_{Y} \mathrm{~d} Y=H_{\mathrm{c}} L \int_{0}^{1} h_{y} \mathrm{~d} y=H_{\mathrm{c}} L \mathcal{V} \\
\Phi=\int_{0}^{l_{1}} B_{Y} \mathrm{~d} X=\mu_{0} H_{\mathrm{c}} L \int_{0}^{\lambda} \mu_{\mathrm{r}} h_{y} \mathrm{~d} x=\mu_{0} H_{\mathrm{c}} L \mathcal{F} .
\end{gathered}
$$

Les courbes $\mathcal{F}(\mathcal{Y})$ fournies par l'ordinateur donnent ainsi directement les fonctions $\Phi(I)$ de la machine. Celles-ci permettent donc d'accéder au travail $W$ par cycle, effectué par deux demi-plots fixe et mobile) dans un volume $l_{1} \times L \times 1$

$$
W=\int_{\text {cycle }} I \mathrm{~d} \Phi=\mu_{0} H_{\mathrm{c}}^{2} L^{2} \int_{1, \lambda} \mathcal{\mathrm { d }} \mathfrak{F} .
$$

Le travail par unité de volume utile, est donc :

$$
W_{0}=\frac{W}{L \times l_{1}}=\frac{\mu_{0} H_{\mathrm{c}}^{2}}{\lambda} \int_{1, \lambda} \vartheta \mathrm{d} \mathcal{F} .
$$

La puissance volumique $P$ peut s'exprimer alors à l'aide de la vitesse $v$ de défilement des plots ou de la fréquence $f$ de parcours des cycles. On a :

$$
P=W_{0} f=W_{0} \frac{v}{\lambda L}=\frac{\mu_{0} H_{\mathrm{c}}^{2}}{\lambda^{2}} \frac{v}{L} \int_{1, \lambda} v \mathrm{~d} \mathcal{F}
$$

que nous pouvons écrire :

$$
P=\mu_{0} H_{\mathrm{c}}^{2} \frac{v}{L} A \operatorname{avec} A=\frac{\int_{1, \lambda} \mathcal{V} \mathrm{d} \mathcal{F}}{\lambda^{2}} .
$$

Les résultats du calcul concernent donc la fonction $A$. Celle-ci possède une signification physique intrinsèque de première importance : $A$, nombre sans dimension, est directement lié à la pression magnétique tangentielle $P_{\mathrm{T}}$, moyenne sur le cycle. On recueille, en effet, dans un volume $\lambda L, L, 1$ pour un cycle de travail

$$
W=\mu_{0} H_{\mathrm{c}}^{2} L^{2}\left(A \lambda^{2}\right)=\left[P_{\mathrm{T}}(\lambda . L .1)\right] \lambda L
$$

ce qui donne

$$
P_{\mathrm{T}}=\mu_{0} H_{\mathrm{c}}^{2} A
$$

Les dernières expressions de $P$ et $P_{\mathrm{T}}$ montrent que :

- l'induction de saturation du matériau (proportionnelle à $H_{\mathrm{c}}$ ) doit être aussi élevée que possible,

- l'épaisseur des disques, peu différente de $L$, doit être aussi faible que possible,

- la fonction $A(e, \lambda, s, t, \mathcal{Y})$ doit être élevée, afin d'optimiser $P$ et $P_{\mathrm{T}}$.

Pour ceci, il faut que les courbes $\mathfrak{F}(\mathcal{Y})$ de conjonction et opposition soient assez éloignées l'une de l'autre.

3. Les plots trapézoïdaux. - 3.1 LE CHOIX DE PARAMÈTRES. - Il est évident qu'un calcul systématique de ce type de plots est très long. De l'étude préliminaire [4] on peut dégager les conclusions suivantes :

- les plots trapézoïdaux dont l'extrémité est plus large que l'embase $(t>1)$ présentent des performances moins bonnes que celles des plots rectangulaires,

- le gain de performance des plots trapézoïdaux est obtenu pour $t<1$ et lorsque ces derniers sont très saturés. Le calcul doit aussi être fait avec des valeurs de $\mathcal{V}$ dépassant le coude de saturation.

Pour l'exploitation numérique, nous avons alors choisi les paramètres de forme suivants :

$$
\begin{aligned}
e & =0,02 ; 0,04 ; 0,10 \\
\lambda & =1 ; 2 ; 4 \\
s & =0,3 ; 0,4 ; 0,5 ; 0,6 \\
t & =0,5 ; 0,75 ; 1 .
\end{aligned}
$$

En ce qui concerne la valeur de $\mathcal{V}$, elle varie de 10 à 200 selon le cas traité.

Le calcul a été effectué pour toute combinaison de ces paramètres. Nous estimons que, dans la pratique, ces valeurs couvrent toute la gamme de machines constructibles. Rappelons que, pour tout $e$ et $\lambda$, $s=0,4$ représente une configuration très performante, et proche de l'optimum [3] pour les plots rectangulaires $(t=1)$. Ces configurations serviront de valeur de référence dans l'analyse qui suit.

3.2 ANALYSE DES RÉSUltATS OBTENUS. 3.2.1. Comparaison avec les plots rectangulaires. Sur les figures $4 a$ et $4 b$ nous avons tracé les courbes $\mathcal{F}(\mathcal{Y})$ de deux configurations de plots caractérisées par $\lambda=1, e=0,04$. La première correspond à un plot rectangulaire de référence $(s=0,4)$. La seconde correspond à un plot trapézoïdal avec $s=0,6$ et $t=0,5$. (Nous verrons par la suite que cette configuration est très proche de l'optimum.) On constate que, en régime linéaire, les courbes $\mathfrak{F}(\mathcal{U})$ (en conjonction et en opposition) appartenant aux figures $4 a$ et $4 b$ ont des pentes pratiquement identiques. Lorsque la saturation intervient, il apparaît une différence considérable entre les écartements des courbes $\mathfrak{F}(\mathcal{Y})$ des deux configurations. 


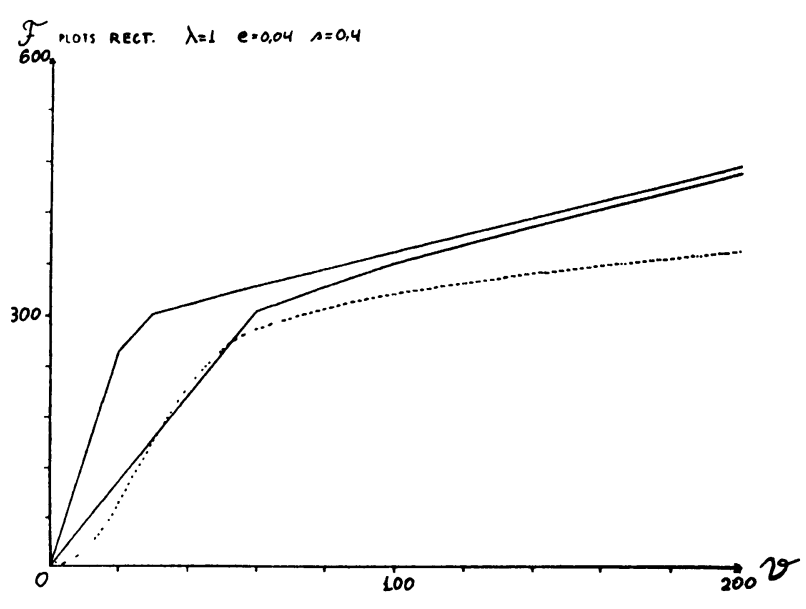

Fig. 4a. - Courbes de flux pour le plot rectangulaire.

[Rectangular slot flux curves.]

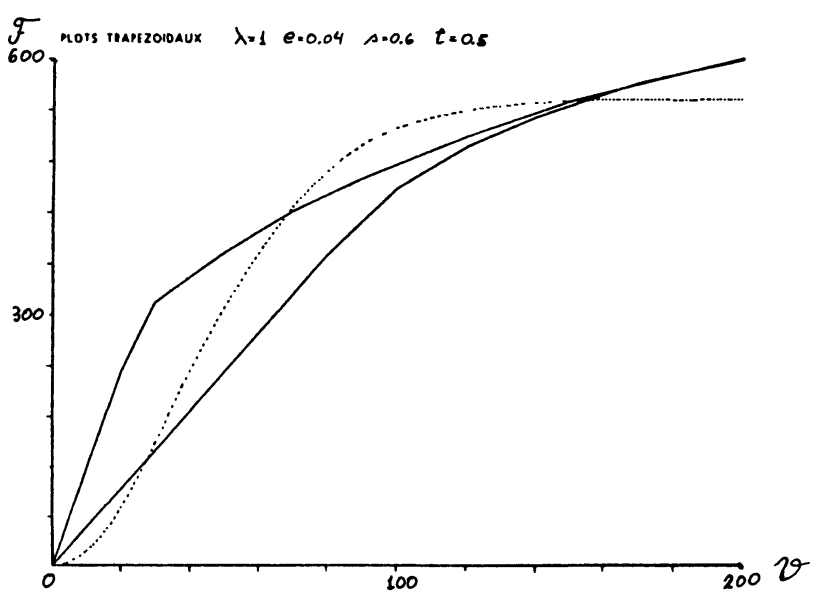

Fig. 4b. - Courbes de flux pour le plot trapézoïdal.

[Trapezoidal slot flux curves.]

Ce phénomène est expliqué de façon qualitative par les remarques suivantes :

- en conjonction, le plot trapézoïdal $(S=0,6)$ ayant une plus grande masse de fer que le plot rectangulaire $(s=0,4)$, sature à des valeurs plus élevées de flux,

- en opposition, l'inclinaison des plots assure un éloignement moyen important entre les plots intérieur et supérieur et la réluctance du domaine reste très proche de celle obtenue avec les plots rectangulaires définis plus haut. La surface comprise entre les courbes $\mathscr{F}(\mho)$ et la droite $\mho=$ Cte, est donc plus élevée avec les plots trapézoïdaux. Les courbes en traits pointillés des figures $4 a$ et $4 b$ sont proportionnelles à ces surfaces. Elles représentent donc, aussi, la pression tangentielle moyenne $P_{\mathrm{T}}$.

On voit qu'en régime linéaire, les courbes sont presque confondues, mais qu'à partir des valeurs de $v$ voisines de 100 , la courbe correspondant au plot trapézoïdal dépasse de plus de $55 \%$ celle du plot rectangulaire.
Sur les figures $5 a, 5 b, 5 c, 5 d$, on montre les lignes de champ obtenues à partir du calcul, pour une configuration de plots très performante. On remarque que la dimension au niveau de l'entrefer $\left(l_{3}\right)$ est proche de celle utilisée pour un plot rectangulaire voisin de l'optimum $(s=0,42)$.

Notons à propos de ces cartes de champ, que leur forme approximative est due au fait que le programme utilisé pour les tracés fonctionne avec un maillage régulier dans le sens horizontal. Nous avons, alors, dû représenter le côté incliné des plots par une succession de marches d'escaliers. Cette condition restrictive n'affecte que le tracé des lignes de champ, car, en ce qui concerne le calcul proprement dit, les côtés des éléments peuvent être inclinés à volonté. Les résultats

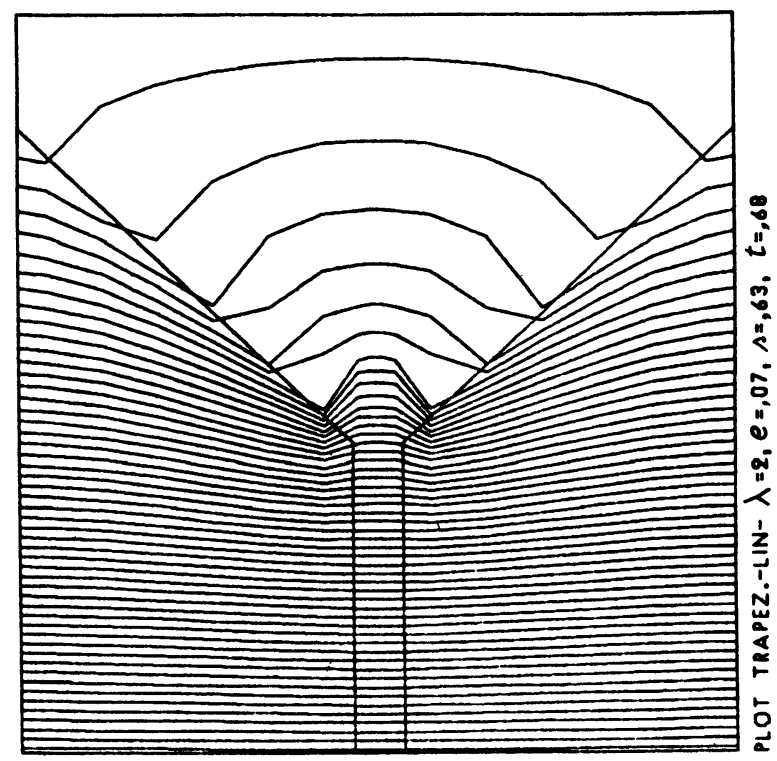

a)

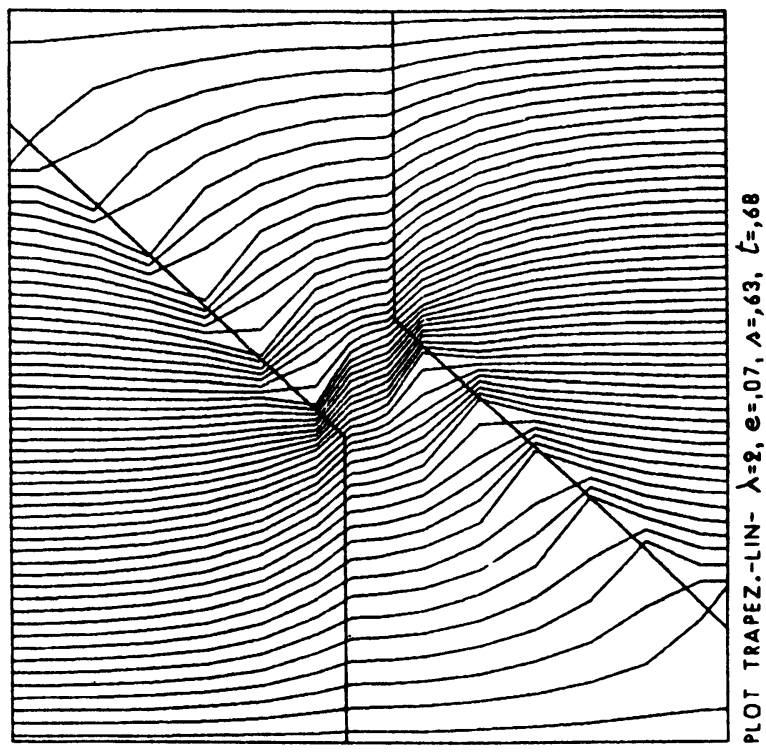

b)

Figs. $5 a, b, c, d$. - Courbes de flux à différents régimes de saturation.

[Flux distribution at differents saturation regimes.] 


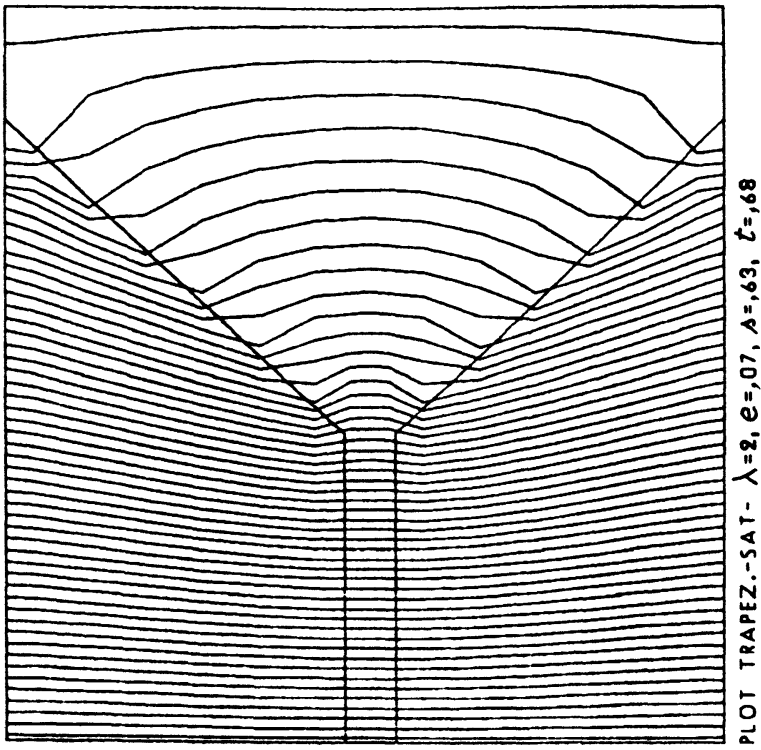

c)

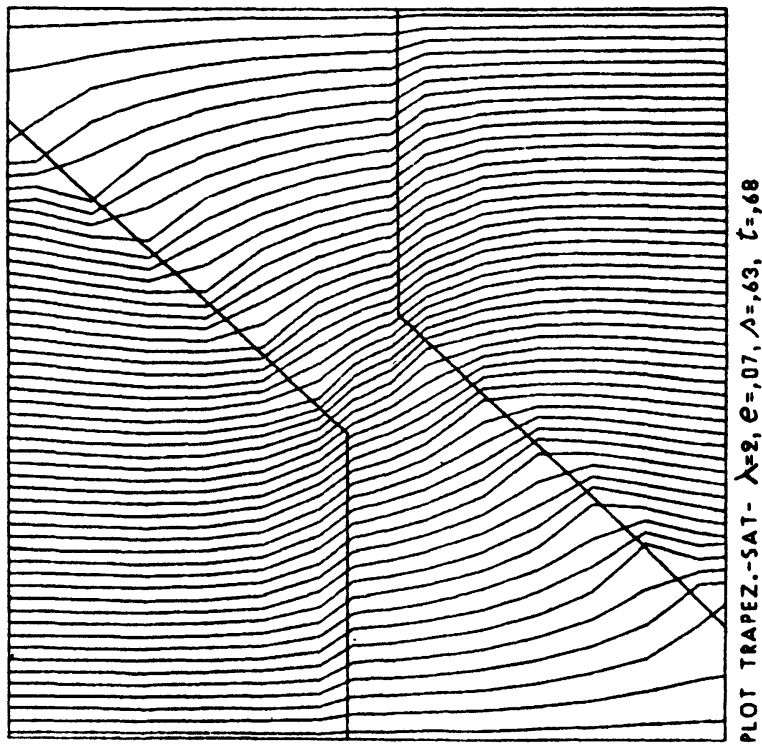

d)

numériques fournis sont donc plus proches de la réalité que les tracés présentés.

3.2.2 Résultats obtenus. - Nous avons sur les figures $6 a, 6 b$ et $6 c$ les courbes de pression tangentielles pour :

$$
\begin{aligned}
\lambda & =1 ; 2 ; 4 \\
e & =0,04 \\
s & =0,4 ; 0,5 ; 0,6 \\
t & =0,5 ; 0,75 ; 1 .
\end{aligned}
$$

Pour $s=0,3$ les performances des plots rectangulaires et trapézoïdaux sont très proches. Les plots trapézoïdaux ne sont avantageux que lorsqu'on travaille à des régimes saturés. Ainsi, on observe sur la figure 6 que, pour $\lambda=1$, on doit utiliser au moins, une valeur voisine de 100 ; pour $\lambda=2$ cette valeur est estimée aux alentours de 140 . Au-delà de 140 , le cou-
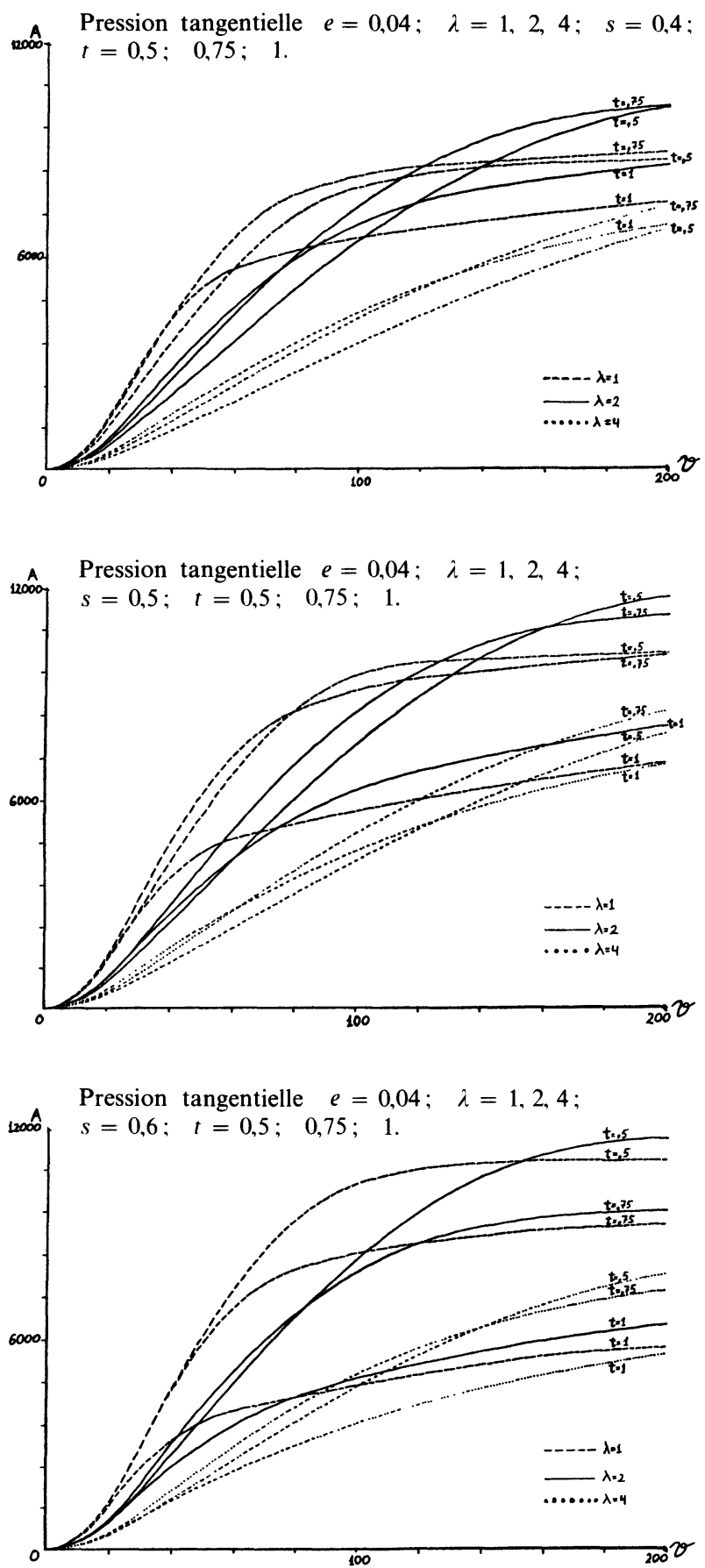

Figs. $6 a, b, c .-$ Pression magnétique tangentielle moyenne réduite en fonction de la DDP et de paramètres de formes.

[« Normalized " average tangential magnetic pressure us a function of DDP and design parameters.]

rant passant dans le bobinage est trop important. Pour que ces courbes soient extrapolables pour d'autres valeurs d'entrefer, nous donnons sur les figures $7 a$ et $7 b$ les courbes $A(e)$ pour deux valeurs caractéristiques de $U$. Rappelons que pour $t=1$ on peut se reporter aux références [3] et [4].

3.2.3 Formes optimales. - A partir des courbes $A(\mathcal{U})$ nous avons tracé des courbes $A(s), A(t)$ à $U, \lambda$ 

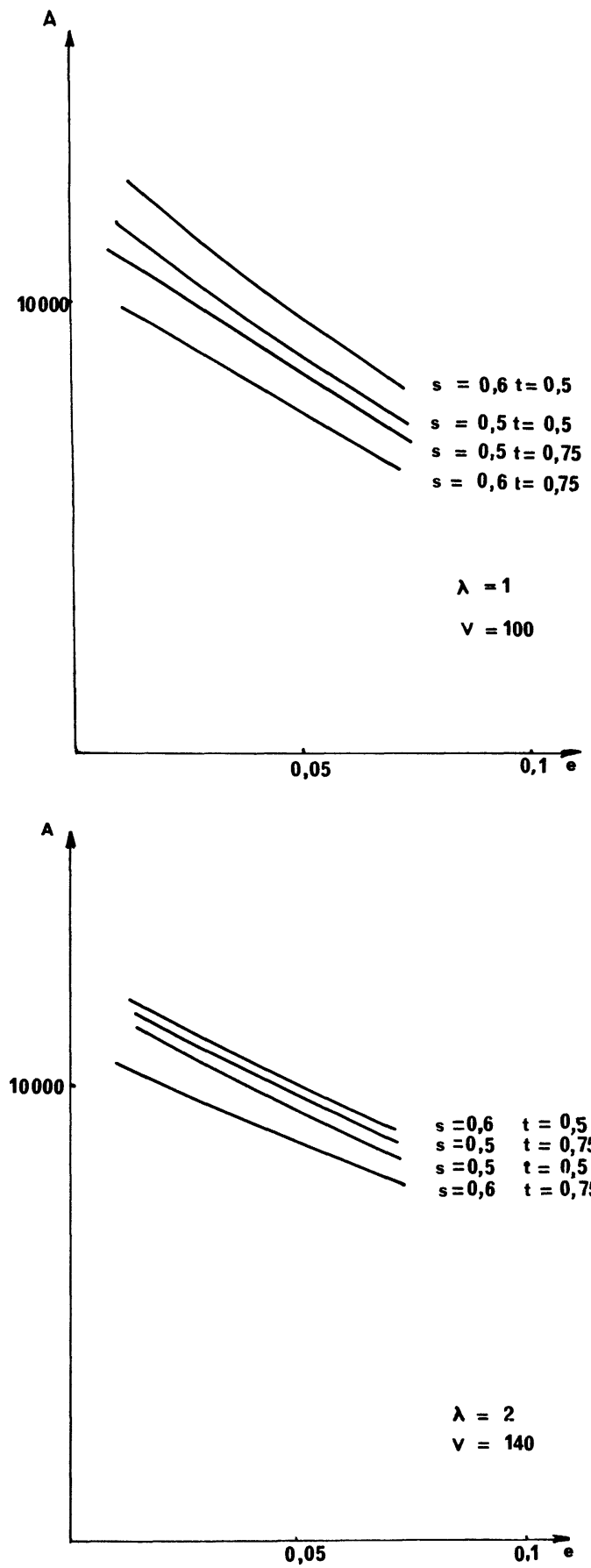

Figs. 7a, b. - Pression tangentielle en fonction de « $e$ ».

[Tangential pressure us a function of « $e »$.]

et $e$ constantes, ce qui nous permet de dégager des valeurs optimales de $s$ et $t$.

Malgré une difficulté d'analyse, due au grand nombre de paramètres, nous dégageons avec une bonne précision, les points essentiels suivants :

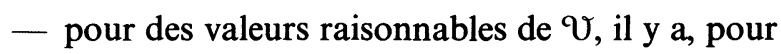
chaque, $s$, un $t$ optimum, et ceci quels que soient $e$ et $\lambda$. La plage de valeurs performantes est :

$$
\begin{aligned}
& s \in[0,5 ; 0,64] \\
& t \in[0,53 ; 0,60] .
\end{aligned}
$$

Les combinaisons de $s$ et de $t$, telles que $l_{3} / l_{1}=s \times$ $t=0,34$ assurent de très bonnes performances. Parmi ces combinaisons, la meilleure est obtenue avec le couple :

$$
s=0,6 \text { et } t=0,57 .
$$

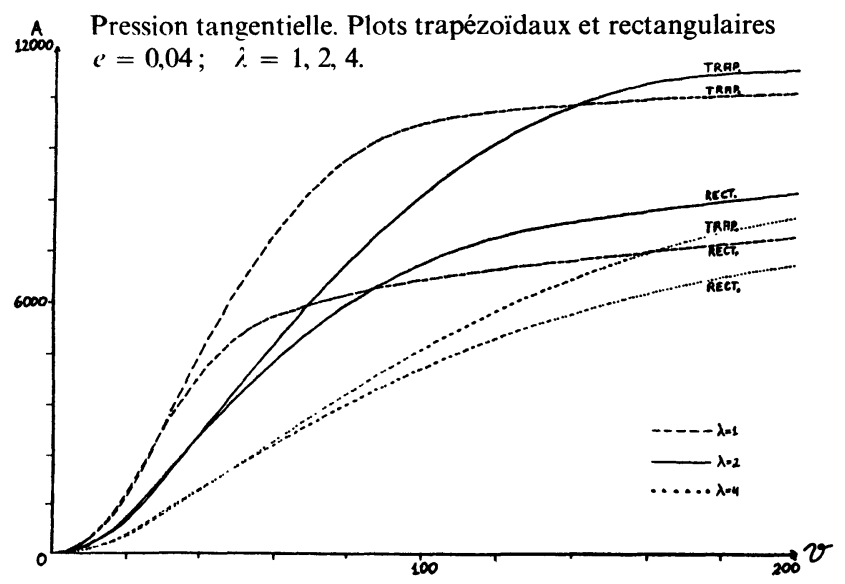

Fig. 8. - Pression tangentielle pour les domaines optimaux.

[Tangential pressure of optimals elementary domains.]

Nous présentons sur la figure 8 les pressions tangentielles des configurations optimales des plots rectangulaires et trapézoïdaux. On voit que plus $\lambda$ est petit, plus le gain est important et ceci pour des valeurs moins élevées de $\mho$. Sur la figure 9, nous avons l'inclinaison $\alpha$ (Fig. $2 c$ ) en fonction de $\lambda$, lorsque $t=0,57$. On remarque que lorsque $\lambda$ augmente, le plot doit être plus incliné.

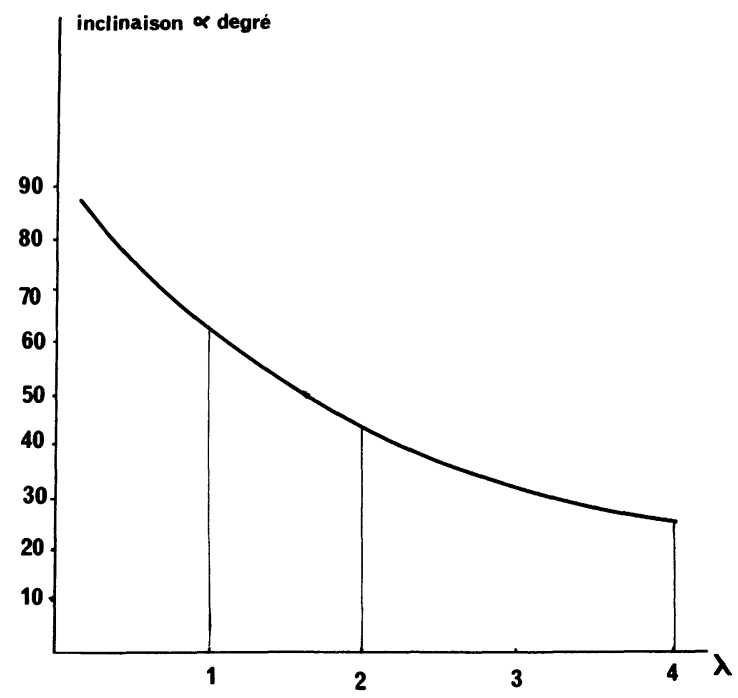

Fig. 9. - Inclinaison du plot en fonction de « $\lambda »$.

[Slot inclinaison us a fonction of $« \lambda »$.]

4. Conclusion. - L'étude sur les plots trapézoïdaux présentée ci-dessus complète celle faite auparavant sur les plots rectangulaires. Les performances de plots 
trapézoïdaux y sont fournies, en régimes linéaire et saturé. Nous en avons dégagé les formes optimales pour ce type de plots.

Comme résultat principal, nous montrons que, en travaillant à des régimes plus saturés, ce type de plot peut fournir une puissance volumique plus importante que celle des plots rectangulaires. Ceci est lié à un passage de flux magnétique plus important à travers du domaine. En ce qui concerne la conception de la machine, ce phénomène peut se traduire de la façon suivante : pour un même nombre de disques et pour un même entrefer, une machine à plots trapézoïdaux peut délivrer, par rapport à une machine à plots rectangulaires, un couple moyen plus important, mais cette amélioration est accompagnée d'un surdimensionnement de bobinages inducteurs et des circuits de retour du flux magnétique. Par conséquent, seule une étude complète de la machine qu'on désire construire permettra de dégager les compromis optimaux.

On remarquera finalement que cette étude est applicable non seulement aux machines à disques imbriqués, mais à toute machine à réluctance variable dont le fonctionnement peut être caractérisé par un cycle flux ampère tours analogue.

\section{Bibliographie}

[1] Rioux, C., Théorie générale comparative des machines électriques établie à partir des équations du champ électromagnétique. Revue Gén. Electr. 79 (1970) 415.

[2] Rioux, C., Aspects préliminaires de la théorie des machines électriques comportant des matériaux ferromagnétiques. Revue Phys. Appl. 15 (1980).

[3] Bastos, J. P., Goyet, R., Lucidarme, J., Performances intrinsèques des machines à réluctance variable à disques imbriqués. Revue Phys. Appl. 15 (1980).

[4] Bastos, J. P., Calcul des performances intrinsèques des machines à réluctance variable polydiscoïdes par une méthode d'éléments finis. Thèse de docteuringénieur (soutenue 5/1980).
[5] ZIENKIEWICS, The finite elements method in engineering sciences (Mc Graw Hill).

[6] Norrie, DeVRIES, An introduction to finite elements analysis (Academic Press, New York, San Francisco, London).

[7] Bastos, J. P., Jablon, C., Quichaud, G., RiouxDAMIDAU, F., Modélisation magnétique et numérique par éléments finis de feuilletages magnétiques. Revue Phys. Appl. 15 (1980).

[8] Goyet, R., Contribution à l'étude des machines à réluctance variable à disques imbriqués. Thèse de Doctorat d'Etat (soutenue juin 1981). 